\title{
Electrochemical Detection of Free-Chlorine in Water Samples Facili- tated by in-situ pH Control using Interdigitated Microelectrodes
}

\author{
Ian Seymour, ${ }^{\mathrm{a}, \mathrm{b}}$ Benjamin O’Sullivan, ${ }^{\mathrm{a}}$ Pierre Lovera, ${ }^{\mathrm{a}}$ James F. Rohan, ${ }^{\mathrm{b}}$ and Alan O'Riordan ${ }^{\mathrm{a}}$ \\ a Nanotechnology Group, Tyndall National Institute, Cork, Ireland. E-mail: alan.oriordan@tyndall.ie \\ b Electrochemical Materials and Energy Group, Tyndall National Institute, Cork, Ireland. E-mail: james.rohan@tyn- \\ dall.ie \\ KEYWORDS Chlorine sensing, Water quality monitoring, In situ pH control, Amperometric Sensor, Interdigitated \\ array, Hypochlorous acid
}

\begin{abstract}
Residual free-chlorine concentration in water supplies is a key metric studied to ensure disinfection. High residual chlorine concentrations lead to unpleasant odours and tastes, while low concentrations may lead to inadequate disinfection. The concentration is most commonly monitored using colorimetric techniques which require additional reagents. Electrochemical analysis offers the possibility for in-line analysis without the need for additional reagents. Electrochemical-based detection of chlorine is influenced by the solution $\mathrm{pH}$, which defines the particular chlorine ionic species present in solution. As such, controlling the $\mathrm{pH}$ is essential to enable electrochemical based detection of residual chlorine in water. To this end, we explore the application of solid state interdigitated electrodes to tailor the in-situ $\mathrm{pH}$ of a solution while simultaneously detecting free-chlorine. Finite element simulations and subsequent electrochemical characterization, using gold interdigitated microelectrode arrays, were employed to explore the feasibility of an in-situ pH control approach. In practice, the approach converted residual chlorine from an initial mixture of two species (hypochlorous acid and hypochlorite ion), to one species (hypochlorous acid). Chlorine detection was shown in water samples using this exploratory method, resulting in a two-fold increase in signal response, compared to measurements without $\mathrm{pH}$ control. Finally, tap water samples were measured using the in-situ $\mathrm{pH}$ control method and the results showed excellent correlation (within experimental error) with a commercial instrument, demonstrating the efficacy of the developed technique. This work establishes the possibility of deploying an electrochemical based reagent-free, in-line chlorine sensor required for water distribution networks.
\end{abstract}

\section{1 - Introduction}

Chlorine disinfection steps are employed for both potable and non-potable water systems and, in each case, knowing the concentration of residual chlorine at the end of the distribution systems is a legal requirement. Residual chlorine is defined as the sum of the concentrations of both chlorine species, hypochlorous acid $(\mathrm{HOCl})$ and hypochlorite $\left(\mathrm{OCl}^{-}\right){ }^{1-3}$ Disinfection processes typically involve bubbling chlorine gas, or adding a salt such as sodium hypochlorite $(\mathrm{NaOCl})$ to the water, Both processes result in the formation of hypochlorous acid as shown by the reaction schemes: 4

$$
\begin{gathered}
\mathrm{Cl}_{2}+\mathrm{H}_{2} \mathrm{O} \leftrightarrow \mathrm{HOCl}+\mathrm{HCl} \\
\mathrm{NaOCl}+\mathrm{H}_{2} \mathrm{O} \leftrightarrow \mathrm{HOCl}+\mathrm{NaOH}
\end{gathered}
$$

The concentration of residual chlorine in a water system must be carefully monitored since too low a value may result in ineffective disinfection and pathogens remaining in the system, while too high may lead to reactions with organic compounds forming, e.g., trihalomethanes, which have been linked to cancer, ${ }^{5}$ respiratory problems ${ }^{6}$ and other adverse health effects. ${ }^{7}$ As a result, the WHO has guidelines that specify the appropriate concentrations of chlorine in drinking water. ${ }^{8}$

Residual chlorine typically exists as a mixture of $\mathrm{HOCl}$ and $\mathrm{OCl}^{-}$, the exact ionic ratio being dependent on solution $\mathrm{pH}$. Drinking water has an acceptable $\mathrm{pH}$ range of 6.5 to 9.5. ${ }^{9}$ While this relatively broad $\mathrm{pH}$ range is suitable for human consumption, it can greatly affect the signal output from a sensor. In this $\mathrm{pH}$ range the chlorine ion ratio can switch from predominantly $\mathrm{HOCl}$ to predominantly $\mathrm{OCl}^{-}$ species; which have very different reactivities at different sensors. ${ }^{10}$ The most common method of detecting residual chlorine is by a colorimetric technique using N,N-diethylp-phenylenediamine (DPD). This involves the reaction between the amine group of DPD and chlorine which produces a pink coloured compound. ${ }^{11}$ Chlorine concentration can then be quantified based on the colour intensity. Similar methods have been developed based on: fluorescence, chemiluminescence and other colorimetric techniques that show high selectivity with limits of detection in the ppb range. ${ }^{1-3}$, 12-14 Although some of these methods mitigate the issue of $\mathrm{pH}$ variation, they can be difficult to 
deploy in-line as required in water distribution networks. They also typically require the addition of a "reagent", which in most cases is not reusable. In contrast, it is well known that electrochemical methods permit detection of other analytes without the need for additional reagents, for example, in medtech ${ }^{15,16}$ and environmental sectors. ${ }^{17}$

Free-chlorine sensors have been described in the literature employing cyclic voltammetry, ${ }^{18-20}$ linear sweep voltammetry, ${ }^{21,22}$ and chronoamperometry ${ }^{23-28}$ as detection methods. Chlorine concentration is probed by measuring the reduction of either hypochlorous acid or hypochlorite according to the following reactions: ${ }^{21}$

$$
\begin{gathered}
\mathrm{HClO}+\mathrm{H}^{+}+2 e^{-} \rightarrow \mathrm{Cl}^{-}+\mathrm{H}_{2} \mathrm{O} \\
\mathrm{ClO}^{-}+\mathrm{H}_{2} \mathrm{O}+2 e^{-} \rightarrow \mathrm{Cl}^{-}+2 \mathrm{OH}^{-}
\end{gathered}
$$

However, conventional electrochemical measurement of free-chlorine in water faces two main challenges. Firstly, as described above, the ratio of the chlorine reactants present in a measuring environment is $\mathrm{pH}$ sensitive. While electrochemical methods can work well in highly controlled $\mathrm{pH}$ environments, with water, the $\mathrm{pH}$ is likely to vary considerably. The second challenge is that hypochlorite is the dominant species at near neutral $\mathrm{pH}$ 's, and electrochemical reduction of hypochlorite occurs in the same potential region as dissolved oxygen. 2930 Consequently, dissolved oxygen is a ubiquitous interferent limiting the effectiveness of electrochemical sensors. ${ }^{26}$ By contrast, hypochlorous acid $(\mathrm{HOCl})$ reduction occurs around o.3 V (versus a saturated calomel electrode, SCE) outside of the oxygen reduction potential window. Thus, novel solutions are required to mitigate the effects of $\mathrm{pH}$ variability when using electrochemical based detection methods.

Prior work has shown that $\mathrm{pH}$ can be controlled in-situ using a rotating ring disc electrode arrangement. Hydrolysis of water produced protons at an anode ring which diffused to the sensing disc electrode lowering the $\mathrm{pH}$ in-situ, compared to the bulk solution and enabled direct detection of mercury. ${ }^{31}$. In acidic conditions, water is split by the following mechanism: ${ }^{2}$

$$
\begin{gathered}
\text { Cathode: } 2 \mathrm{H}^{+}+2 e^{-} \rightarrow \mathrm{H}_{2} \\
\text { Anode: } 2 \mathrm{H}_{2} \mathrm{O} \rightarrow \mathrm{O}_{2}+4 H^{+}+4 e^{-}
\end{gathered}
$$

In basic conditions, the mechanism becomes:

$$
\begin{gathered}
\text { Cathode: } 2 \mathrm{H}_{2} \mathrm{O}+2 e^{-} \rightarrow 2 \mathrm{OH}^{-}+\mathrm{H}_{2} \\
\text { Anode: } 4 \mathrm{OH}^{-} \rightarrow \mathrm{O}_{2}+2 \mathrm{H}_{2} \mathrm{O}+4 e^{-}
\end{gathered}
$$

An acidic medium can be made more basic by consumption of protons in a reduction reaction, or alternatively more acidic by production of protons in an oxidation reaction (depending on the potential imposed at an electrode). It is important to note that the $\mathrm{pH}$ change is strongest close to the electrode and diminishes with increasing distance from the electrode.

The electrochemical measurements in this work are performed on a generator-collector type device composed of two combs of interdigitated electrode arrays. The working electrodes are spaced $10 \mu \mathrm{m}$ apart while the counter electrode is $1.1 \mathrm{~mm}$ away from the region of interest. By imposing an appropriate potential at one ("protonator") comb of electrodes, a pH change occurs in the local environment that tailors the $\mathrm{pH}$ at the other ("sensor") comb. That comb can then be used to perform sensing in conditions that differ from the bulk solution. It is vital that the counter electrode is relatively well removed spatially from the interdigitated combs. This ensures that the consumption of protons does not occur too close to the sensing electrode, which would inevitably prevent $\mathrm{pH}$ control. Using this approach, a local environment is created that is more acidic (or basic) than the bulk conditions. We apply this method to sensing of free-chlorine in the hypochlorous acid ionic form by electrochemically shifting the $\mathrm{pH}$ at a sensor to more acidic conditions. Consequently, at low $\mathrm{pH}$ all freechlorine ions exists as hypochlorous acid, facilitating a more sensitive analysis, that is also removed from the dissolved oxygen interferent reduction potential region. The close spacing of the electrode combs ensures that $\mathrm{pH}$ control is established by the rapid diffusion of protons, so additional acid addition, convection or fluidic forces are not required. Thus, this approach has the potential for deployment as in-line analysis process, required, for example, in water distribution systems

\section{2 - Experimental Section}

\section{1 - Electrode Fabrication:}

Silicon chip based devices were fabricated using methods similar to those described by Dawson et al.33-35 Each chip consisted of two combs of gold working interdigitated electrodes, platinum pseudo reference and gold counter electrodes were also employed on-chip. In brief, chips were designed to interface with external electronics via a microSD port to facilitate facile electrical connection. All of the devices were fabricated on 4-inch silicon wafers bearing a thermally grown $300 \mathrm{~nm}$ silicon dioxide layer. Blanket metal evaporations of Titanium (10 nm) and Gold (100 nm) using a Temescal FC-20oo E-beam evaporator and lift-off technique yields interdigitated microband ( $55 \mu \mathrm{m} \times 1 \mu \mathrm{m} \times$ $60 \mathrm{~nm}$ ) structures with gaps between the combs of 1, 2 and $10 \mu \mathrm{m}$. A second metal evaporation and lift-off process yields the interconnection tracks, contact pads and the gold counter electrode ( $90 \mu \mathrm{m} \times 7 \mathrm{~mm})$. Finally, a third metal evaporation was performed to create the platinum pseudo reference electrode. To prevent unwanted interactions along the connection tracks, silicon nitride, which acts as an insulting layer was deposited by plasma, enhanced chemical vapour deposition. Photolithography and dry etching were utilised to selectively open windows ( 45 $\mu \mathrm{m} \times 100 \mu \mathrm{m})$ in the insulating SiN layer over the microband electrodes for exposure to the electrolyte. Openings 
were also created over the counter and pseudo-reference electrodes and the contact pads. Each device contains six interdigitated electrode (sensors) which are separated by $0.94 \mathrm{~mm}$. Once the sensor fabrication is completed, a wafer was diced into 28 separate chip devices.

A custom made holder cell was fabricated to allow measurement in small electrolyte volumes $(\sim 50 \mu \mathrm{L}$ to $5 \mathrm{mls})$. The cell was constructed from an aluminium base and a Teflon lid. Spring loaded probes (Coda Systems Ltd. PM4J Plain Radius Microprobes) were inserted into the lid in position above the peripheral contact pads, to permit electrical connection to external potientiostats. The cell was assembled with a Viton O-ring embedded in the lid to forma seal around the on-chip electrodes. Viton O-rings were chosen for their chemical resistance. The inner diameter of the O-ring was $7 \mathrm{~mm}$ with a cross section of $1.6 \mathrm{~mm}$ to allow an opening large enough to expose all six sensors, counter and reference electrodes on the device to the electrolyte.

\section{2 - Electrode Characterisation:}

Each chip was inspected using optical microscopy to identify any obvious defects or faults. Prior to any electrochemical characterisation chips were cleaned by immersion in acetone, then iso-propyl alcohol and finally de-ionized water, each for a period of ten minutes. The chips were dried in a flow of nitrogen and placed in the chip holder. Electrochemical analysis was performed using an Autolab Bipotentiostat (MAC80150 with BA Module, Metrohm). Cyclic voltammograms (CV) were performed from o $\mathrm{V}$ to $0.6 \mathrm{~V}$ at $50 \mathrm{mV} / \mathrm{s}$ in $1 \mathrm{mM}$ ferrocene carboxylic acid (FCA, Sigma Aldrich, 97\%). During these scans, the second interdigitated comb of electrodes were held at o V. All electrochemical measurements were recorded versus a SCE.

\section{3 - Buffer preparation and electrode characterisation:}

A series of buffers of differing $\mathrm{pH}$ was used to study the gold oxide reduction reaction. $0.1 \mathrm{M}$ citric acid (Riedel-de Haën, 99.5\% anhydrous) and o.2 M sodium phosphate dibasic (Merck, 99\% anhydrous) were mixed in appropriate ratios to yield buffers with $\mathrm{pH}$ values of 3.6, 4.6 and 7.6, respectively. $0.2 \mathrm{M}$ sodium phosphate dibasic and $0.2 \mathrm{M}$ sodium phosphate monobasic (Sigma Aldrich, 99\%) were mixed to make a $\mathrm{pH} 8.6$ buffer, while o.1 M sodium carbonate (Sigma Aldrich, 99\%) and o.1 M sodium bicarbonate (Sigma Aldrich, 99.5\%) were mixed to yield a $\mathrm{pH}$ 10.8 buffer. Voltametric analysis was performed in each buffer over the potential range o to $1.2 \mathrm{~V}$ (versus SCE) at 50 $\mathrm{mV} / \mathrm{s}$.

\section{4 - pH adjustment in Water Samples:}

Deionised water samples and artificial drinking water (ADW) samples were used to assess the ability of the microarray to control $\mathrm{pH}$. ADW was prepared by dissolving 1 $\mathrm{g}$ of sodium bicarbonate, $0.0654 \mathrm{~g}$ of magnesium sulphate (Sigma Aldrich, 99.5\% anhydrous), $0.3414 \mathrm{~g}$ calcium sulphate dehydrate (Honeywell, 99\%), 0.007 g potassium phosphate dibasic (Fluka, 98\%), potassium phosphate monobasic (Sigma Aldrich, 99\%) and o.o1 g sodium nitrate (Sigma Aldrich, 99\%) in 10 L of deionised water. For in-situ $\mathrm{pH}$ control, voltammograms were performed in both DI and ADW samples by scanning the sensing comb of the IDE array from $0.2 \mathrm{~V}$ to $1.2 \mathrm{~V}$ (versus $\mathrm{SCE}$ ) at $50 \mathrm{mV} / \mathrm{s}$ with the protonator comb biased at $1.65 \mathrm{~V}$ (which is in the oxygen evolution region) to protonate (acidify) the local environment of the sensing electrodes.

\section{5 - Diffusion simulations:}

Diffusion simulations of proton concentration in the vicinity of the protonator electrodes were undertaken according to Fick's second law. A model was designed to simulate generation at and diffusion of protons from the protonator electrodes using finite element analysis (FEA) software, COMSOL Multiphysics 5.3, in line with the galvanostatic model shown by Read et al ${ }^{31}$. The geometry of the model consisted of a $5 \mathrm{~mm}$ square box as the experimental domain, and two sets of interdigitated $1 \mu \mathrm{m}$ wide microband electrodes (14 protonator electrodes and 13 sensing electrodes), separated by $2 \mu \mathrm{m}$. A flux of protons was applied at the surface of the protonators, by applying a fixed anodic current, where the flux was assumed to be proportional to the current applied at the electrodes. The initial $\mathrm{pH}$ value was set to 7 . The proton diffusion coefficient used for the simulation was $9.31 \times 10^{-5} \mathrm{~cm}^{2} \mathrm{~s}^{-1}$.

\section{6 - Detection of Free-Chlorine Without In-situ pH Con- trol:}

Initial scans were performed in various concentrations of free-chlorine in $\mathrm{ADW}$ at $\mathrm{pH}_{3}$ (acidified using $\mathrm{HCl}$ ) to establish the appropriate linear sweep voltammetry (LSV) parameters. Working samples were prepared by diluting Milton Sterilising Fluid (2\% Sodium Hypochlorite) to the required concentrations. The concentration of free-chlorine was measured using a commercial free-chlorine colorimeter (Pocket Colorimeter II 58700-oo with $\mathrm{Cl}_{2}$ Test Kit, DPD method). The samples were acidified using $0.1 \mathrm{M} \mathrm{HCl}$ to ensure the dominant chlorine ion species was hypochlorous acid. LSV potential parameters were determined to be $0.95 \mathrm{~V}$ to $0.2 \mathrm{~V}$ (versus SCE) with a $50 \mathrm{mV} / \mathrm{s}$ scan rate. Scans were also performed using the same LSV parameters at $\mathrm{pH} 8.5$ to establish the detection of Free-chlorine when the sample is a mixture of hypochlorous acid and hypochlorite.

\section{7 - Detection of Free-Chlorine With pH Control:}

The same samples of chlorine in ADW used for the tests at $\mathrm{pH} 8.5$ were used for the experiments using $\mathrm{pH}$ control. The LSV parameters were as before with the addition of a bias of $1.65 \mathrm{~V}$ imposed on the protonator comb of electrodes to acidify the local environment. 


\section{3 - Results and Discussion}

\section{1 - Device Characterisation:}

Devices were fabricated with varying inter-electrode comb spacing (1, 2 and $10 \mu \mathrm{m})$. Each comb of interdigitated electrodes can be addressed separately allowing for generator-collector type sensing applications. Figure 1 (A) shows an image of a silicon chip device which consists of six sensors and on-chip counter and reference electrodes. Each sensor comprised of two interdigitated electrode combs, a protonator and sensor comb, respectively. Figure 1 (B) shows a higher magnification image of a sensor with a $2 \mu \mathrm{m}$ gap between electrode combs. The protonator comb (left hand side) contains 14 electrodes, while the sensor comb (right hand side) has 13 electrodes. The passivation is opened directly over the interdigitated combs of electrodes which is evident by the darker blue coloured rectangular window. This prevents unwanted electrochemical reactions occurring along the interconnection tracks. It is also clear that the electrodes do not touch the opposite side of the array which indicates that there is no electrical short in the device so dual mode generator-collector type electrochemical measurements are possible.

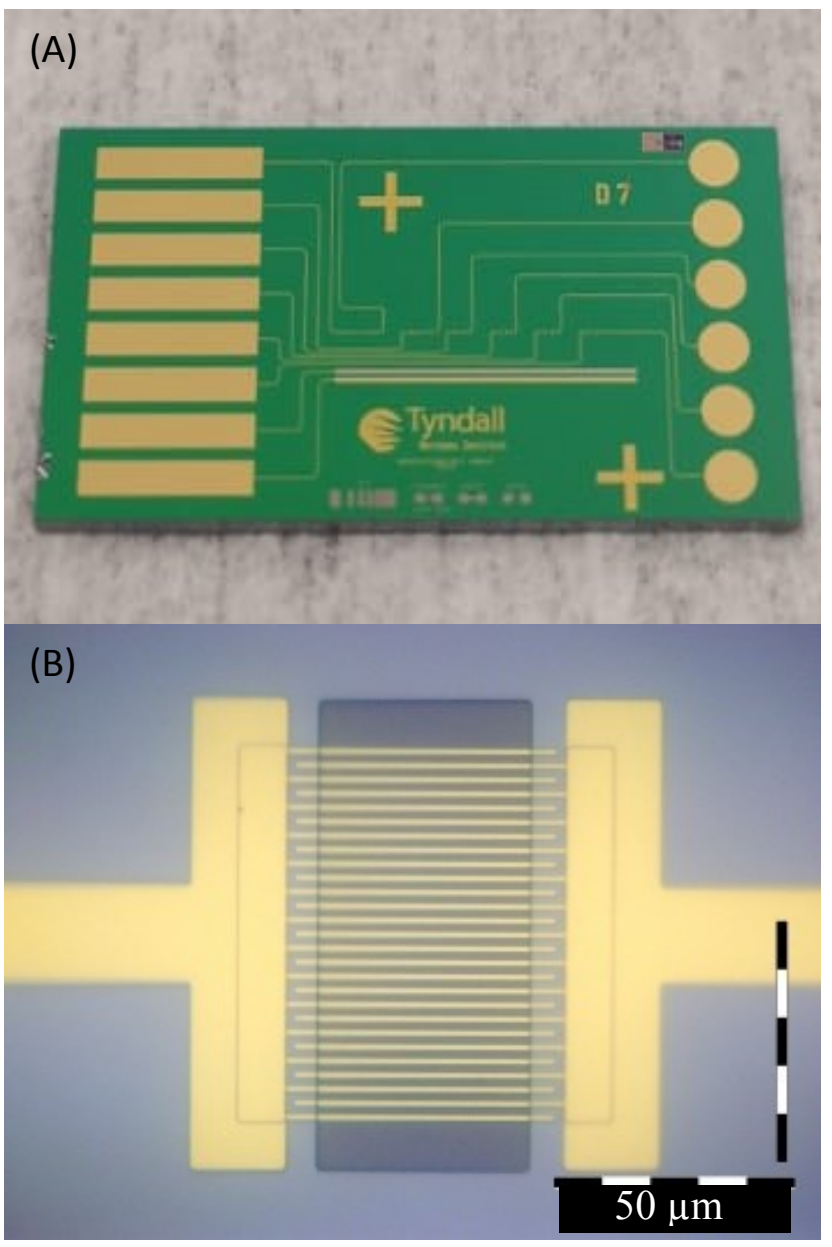

Figure 1: (A) Photograph of the full silicon chip ( $9.68 \mathrm{~mm}$ by $17.42 \mathrm{~mm}$ ) showing contact pads, connection tracks, counter electrode, reference electrodes and sensors. (B) $50 x$ magnification of a single sensor array highlighting where the gold IDEs make contact to the gold pads.

Following on from optical inspection, sensors were electrochemically characterized using FCA. Figure 2 shows a typical scan performed in generator-collector mode. The generator was cycled from o $\mathrm{V}$ to $0.6 \mathrm{~V}$ while the collector was held at o V. The generator comb oxidised the FCA to $\mathrm{FCA}^{+}$. The $\mathrm{FCA}^{+}$species diffused across the gap to the collector electrode, which subsequently reduces it back to FCA. This is a phenomenon known as redox cycling and can be used to boost signals as described by Wahl et al. ${ }^{36}$ The shape and current magnitude seen for the FCA scan in figure 2 was typical of a working electrode array. The voltammogram in Figure 2 exhibits steady-state behavior, for the oxidation of FCA. This is a result of ultra-microelectrodes permitting time independent mass transfer when in the generator-collector mode. As such there is no overlap of diffusion layers, which would cause the array to behave as one larger electrode, diminishing sensitivity. The collection efficiency, which is a ratio of the collector to the generator currents, was determined to be $83.5 \%$, for $2 \mu \mathrm{m}$ gaps. The voltammogram in Figure 2 graph represents three consecutive $\mathrm{CV}$ each showing excellent overlap with the previous scans demonstrating the reproducibility of the sensors. The generator voltammogram exhibited a capacitance current component arising from the fast scan rate applied. However, the collector voltammogram has a much lower capacitative current component as it was held at a constant potential throughout the experiment. Thus it's capacitance dissipated after initial biasing, evidenced by the lack of hysteresis between the forward and reverse sweeps in the collector scan in Figure 2.

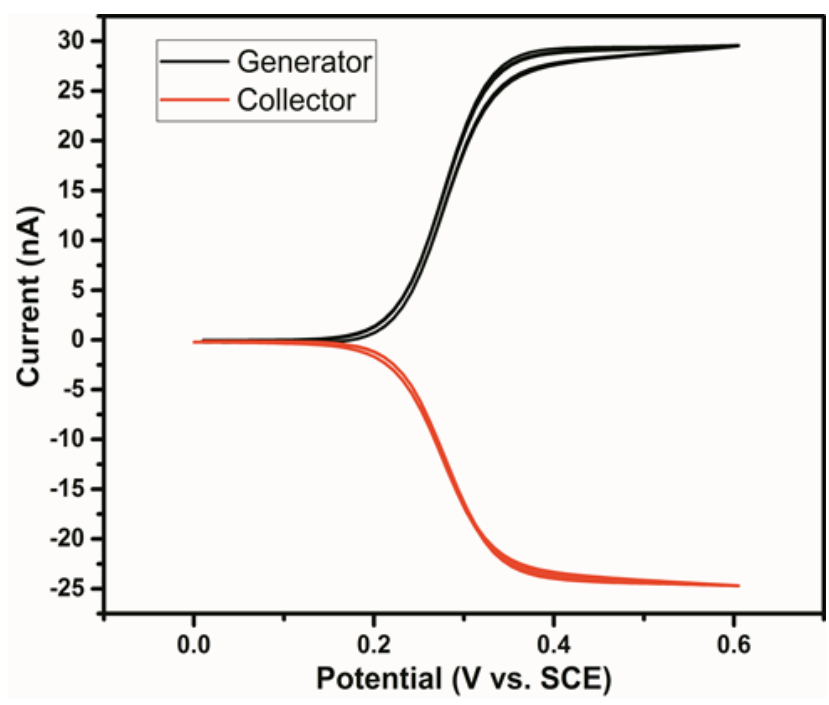

Figure 2: CVs at gold IDEs of $1 \mathrm{mM} \mathrm{FCA} \mathrm{in} 10 \mathrm{mM}$ PBS at a scan rate of $50 \mathrm{mV} / \mathrm{s}$. The generator electrode (black) was cycled between $0 \mathrm{~V}$ and $0.6 \mathrm{~V}$ while the collector (red) was held at $0 \mathrm{~V}$. A current of $30 \mathrm{nA}$ was indicative of a fully working array. This graph shows three consecutive cycles each showing excellent overlap with the previous scan.

\section{2 - pH Dependence of Gold Oxide Reduction Peak:}

The gold oxide reduction peak was used as a probe for the $\mathrm{pH}$ condition of the electrodes. An oxide was formed on a gold electrode by scanning to a sufficiently positive potential, typically around $1.2 \mathrm{~V}$. The electrode was then swept cathodically to the initial potential and the position of the oxide reduction peak was noted. This procedure was 
repeated at different $\mathrm{pH}$ values in a series of buffers to establish the linearity of the technique. Figure 3 (A) shows the oxide reduction peaks for the different buffer solutions. In each case; four replicate scans were performed to determine reproducibility. As expected the oxide peaks shifted to more positive potentials at lower $\mathrm{pH}$ values. The oxide peak potential values for different $\mathrm{pH}$ 's were reproducible (SD of $6.32 \mathrm{E}-4 \mathrm{~V}$ for greatest error) over multiple scans as confirmed by the negligible error bars for the calibration in figure 3 (B) fitted using a linear regression approach. The calibration plot indicates a strong linearity with an $\mathrm{R}^{2}=$ 0.998 .

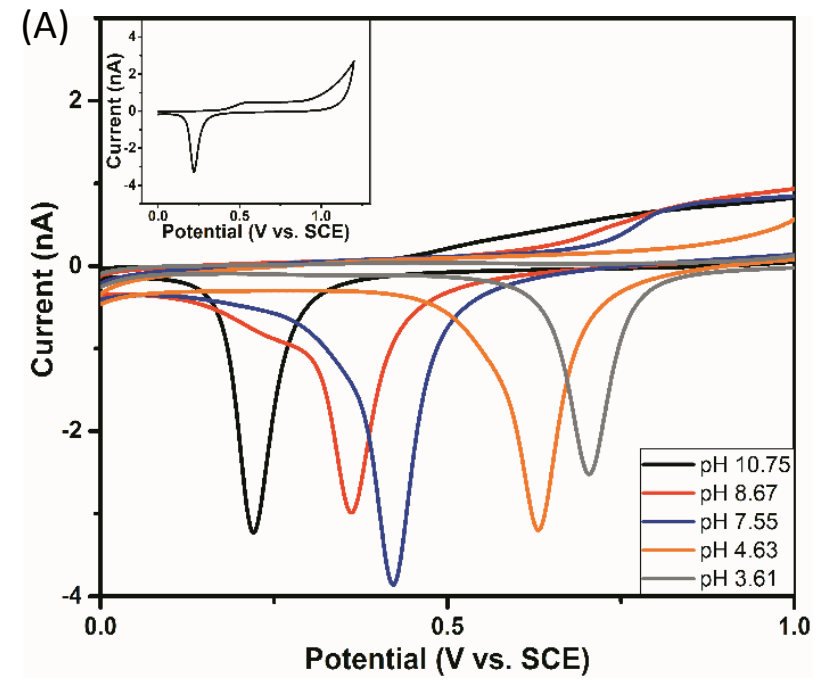

(B)

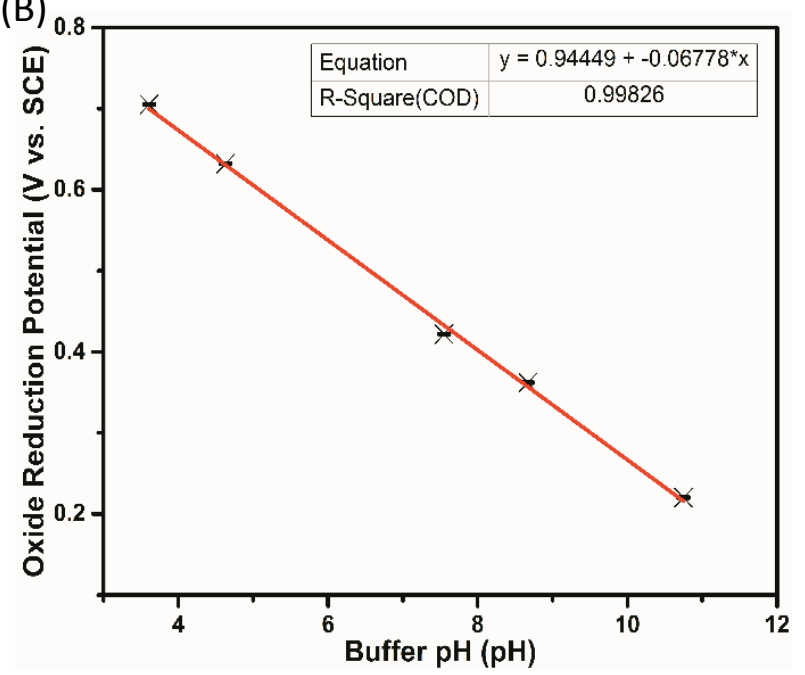

Figure 3: (A) CVs of one electrode comb in a series of $\mathrm{pH}$ buffers from 0 to $1.2 \mathrm{~V}$ at 50 $\mathrm{mV} / \mathrm{s}$. For these scans, the second electrode was left unbiased to prevent any interference. The inset shows the full scan used to take a measurement. (B) Calibration plot of oxide peak reduction potential vs. pH. A linearity fit of 0.998 is determined for this calibration plot.

The oxide approach to $\mathrm{pH}$ analysis shows a sensitivity of $67.78 \mathrm{mV} / \mathrm{pH}$, indicated by the slope of the linear fit. As mentioned, the error was very low for each data point so the error bars are not well defined. The error was calculated as three times the standard deviation between scans, which is used for each calibration.

\section{3 - Simulation of $\mathrm{pH}$ Control at Interdigitated Elec- trodes:}

To determine the viability of the proposed in-situ $\mathrm{pH}$ control method using interdigitated electrodes, a simulation study was performed to elucidate the diffusion of $\mathrm{H}^{+}$ ions away from a protonator electrode with $2 \mu \mathrm{m}$ separations. The simulation model was employed to determine the best electrode configuration for $\mathrm{pH}$ control and to establish some of the conditions necessary to tailor the local $\mathrm{pH}$ to the acidic values required. Figure 4 (A) shows the $\mathrm{pH}$
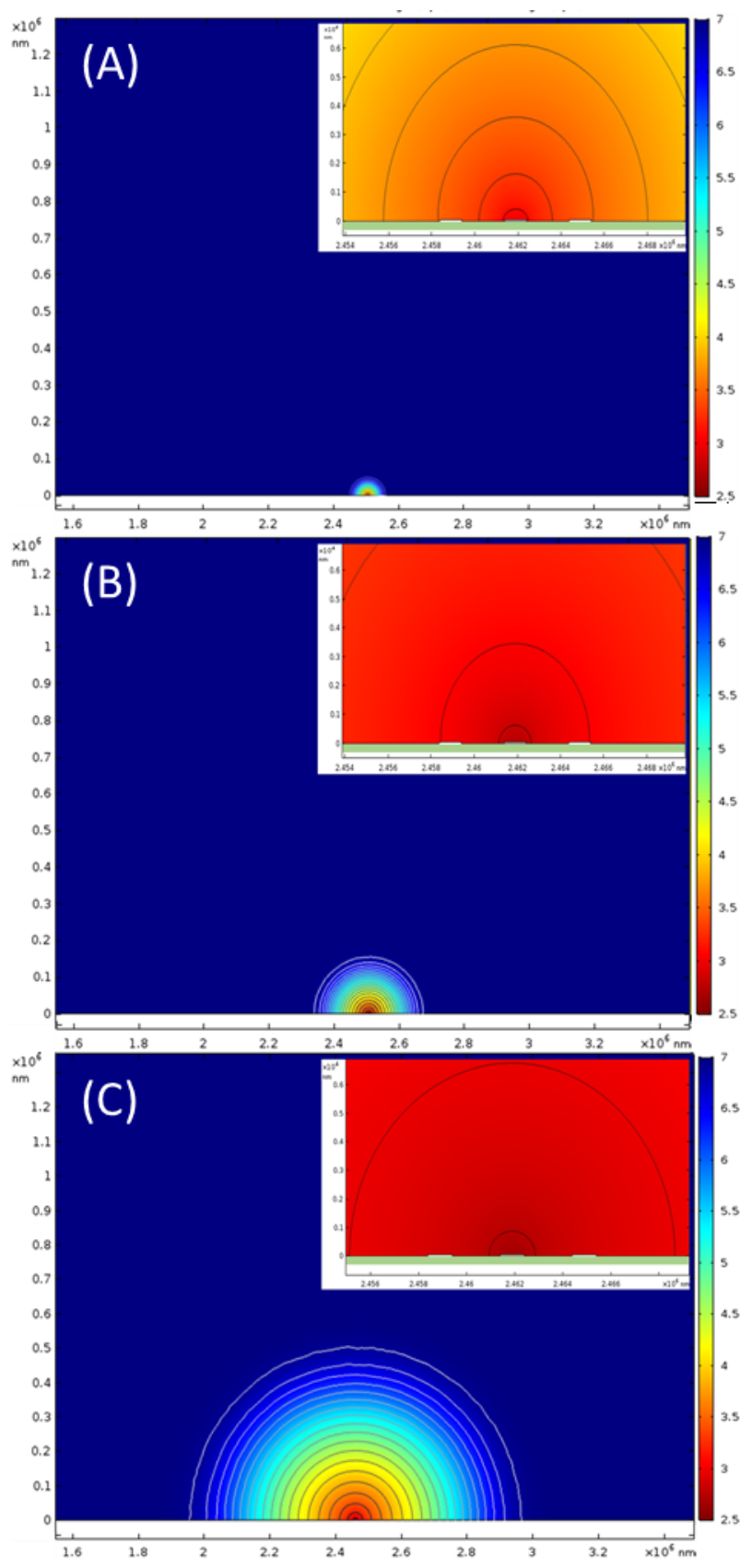

Figure 4: Simulation of an interdigitated array of electrodes with a current of 1 $\mu \mathrm{A}$ applied to the inner electrode. Simulations show the local pH $10 \mathrm{~ms}(\mathrm{~A}), 100$ $\mathrm{ms}$ (B) and $1000 \mathrm{~ms}$ (C) after current imposition. The insets are a higher magnification of the 3 electrodes highlighting the local pH environment. The insets also show the silicon substrate (light green colour). 
in the environment of the electrodes after applying a current of $1 \mu \mathrm{A}$ for $10 \mathrm{~ms}$. The image shows a 2-D cross-section of a $1.2 \mathrm{~mm}$ by $1.8 \mathrm{~mm}$ area through the interdigitated array. It can be seen that although the bulk $\mathrm{pH}$ value remains at its initial value (in this case $\mathrm{pH}$ 7.0), in the locality of the electrodes the $\mathrm{pH}$ has begun to drop from $\mathrm{pH} 7$ to below 5 (see inset - zoom in showing a $6 \mu \mathrm{m}$ by $16 \mu \mathrm{m}$ area around the electrodes). Figure 4 (B) shows the situation $100 \mathrm{~ms}$ after the current bias was applied. The diffusion layer thickness has increased and the local environment at the electrodes has decreased to below $\mathrm{pH}_{4}$. Figure 4 (C) shows the local environment $1 \mathrm{~s}$ after the current bias was applied. The diffusion layer thickness has increased further and the local environment at the electrodes is now at $\mathrm{pH}_{3}$ (Inset). This suggests that within 1 second from switching on the $\mathrm{pH}$ control, the local $\mathrm{pH}$ environment should be sufficiently low to convert all residual chlorine to hypochlorous acid ionic form.

\section{4 - Evaluation of in-situ pH Control of Deionised Water and Artificial Drinking Water:}

As previously discussed, the location of the gold oxide reduction peak was used to determine the parameters required for $\mathrm{pH}$ control in non-buffered matrices as shown in Figure 3. Deionised water samples were used initially to minimize complications arising from buffering capacity and interfering species. The samples were made slightly basic (pH 8.5) using $10 \mathrm{mM} \mathrm{NaOH}$, as water splitting is not efficient at neutral $\mathrm{pH}$. Tests were performed in triplicate where the sensing comb was swept anodically from $0-\mathbf{1 . 2}$ $\mathrm{V}$ while the protonator remained unbiased. A gold oxide was formed on the electrode and subsequently reduced on the cathodic sweep. The location of the reduction peak maximum was observed at $\sim 0.27 \mathrm{~V}$, see Figure 5 (a). The protonator electrode was then biased at a constant positive potential of $1.65 \mathrm{~V}$ (vs. SCE) to induce a local $\mathrm{pH}$ change and the sensor electrode again swept from o -1.2 V. These results are shown in figure $5(\mathrm{~A})$, where the gold oxide reduction peak of the sensor electrode was observed to shift anemically to $0.75 \mathrm{~V}$; a shift of $48 \mathrm{mV}$. Using the data presented in Figure $3(\mathrm{~B})$ it is estimated that the induced $\mathrm{pH}$ change was from $\mathrm{pH} 8.5$ to $\mathrm{pH} 2.9$ at the "sensor" electrode; a drop of $\sim 5 \mathrm{pH}$ units.

Tests were then repeated in ADW to determine the effectiveness of $\mathrm{pH}$ control in real sample conditions. ADW contains significant concentrations of sodium bicarbonate which was expected to buffer the $\mathrm{pH}$ at around 8.5. Using the same parameters as described above, it was found that $\mathrm{pH}$ control was still possible under these buffered conditions, see Figure $5(\mathrm{~B})$. The $\mathrm{pH}$ shift is not as large as for deionised water, which was expected, but it was still sufficient to decrease the $\mathrm{pH}$. The oxide reduction peak in this case appears at $0.68 \mathrm{~V}$, which was estimated to be $\mathrm{pH} 3.8$. While not as acidic as the deionised water sample, this $\mathrm{pH}$ still enables a near total conversion to $\mathrm{HOCl}$. By adjusting the protonator potential further, the $\mathrm{pH}$ change can be tailored to the required value of 3.0.

\section{4 - Free-Chlorine Detection Using pH Control:}

Samples of hypochlorous acid (made by diluting a hypochlorite stock in ADW and acidifying with $10 \mathrm{mM} \mathrm{HCl}$ ) were assessed using the interdigitated electrodes to establish the appropriate detection window. LSV was again used as the detection method and it was found that scanning from $0.95 \mathrm{~V}$ to $0.2 \mathrm{~V}$ at $50 \mathrm{mV} / \mathrm{s}$ was optimal for detection of Free-chlorine. A series of low concentration (0.3-2 ppm) hypochlorous acid standards ( $\mathrm{pH}$ of approximately 8.5) were prepared and their respective concentrations confirmed using a commercial DPD method. Figure 6 (A) shows typical LSV scans obtained at a senor electrode while the protonator electrode remained un-biased. Under these conditions, the Free-chlorine will exist as both hypochlorous acid and hypochlorite, with the latter being the predominant species. Voltammograms recorded for the 0.35 and 0.7 ppm standards overlapped entirely, as the currents associated with each are quite low, and could not be
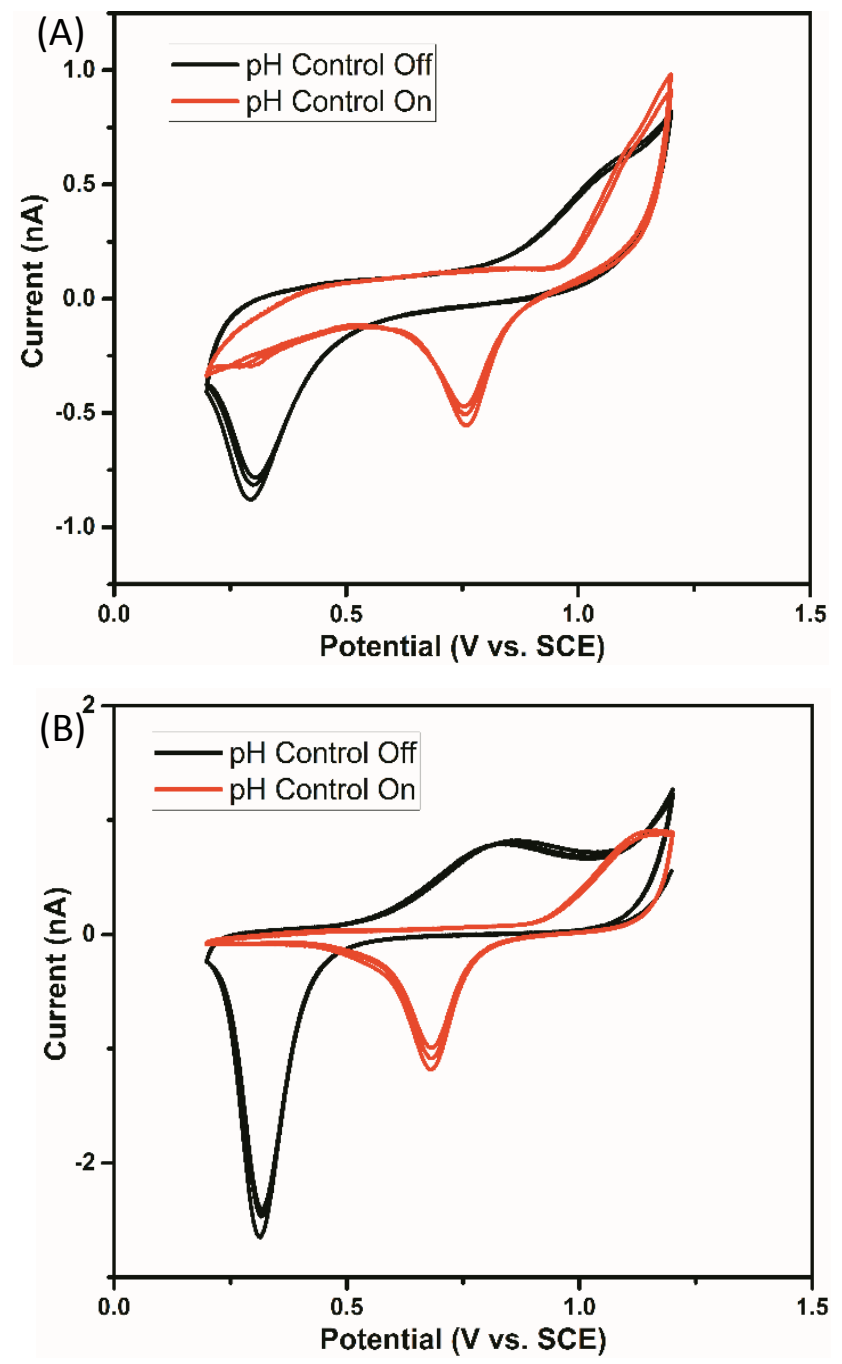

Figure 5: CVs at gold "sensor" IDE's over the range 0.2 to $1.2 \mathrm{~V}$ vs SCE at a sweep rate of $50 \mathrm{mV} / \mathrm{s}$. (A) Deionised water at an Initial pH is 8.5 the oxide reduction peak is seen at approximately $0.27 \mathrm{~V}$ (black). By biasing the second electrode at $1.65 \mathrm{~V}$ the oxide reduction peak shifts to $0.75 \mathrm{~V}$ (red). (B) The same parameters were applied to a sample of ADW 

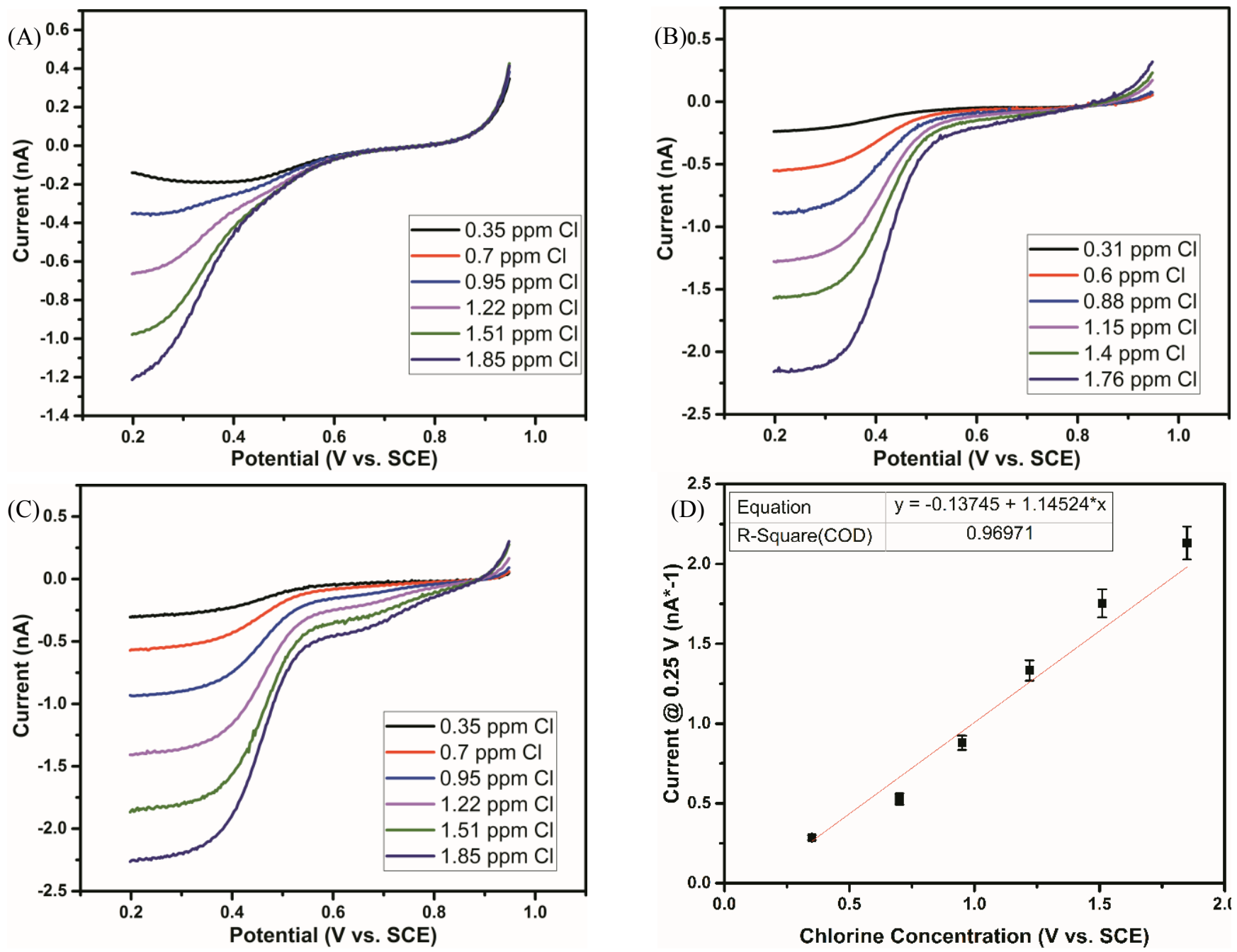

Figure 6: LSVs at gold IDE's over the range 0.95 to $0.2 \mathrm{~V}$ vs SCE at a sweep rate of $50 \mathrm{mV} / \mathrm{s}$. (A) Various concentrations of chlorine, measured by the DPD method in ADW at pH 8 . (B) Various concentrations of chlorine, measured by the DPD method in ADW at $\mathrm{pH}$ 3. (C) Various concentrations of chlorine, measured by the DPD in ADW at pH 8 with the protonator electrode biased at 1.65 V. (D) Calibration plot for the scans shown in (C).

differentiated from each other. A well-defined plateau current for the reduction was not observed making it difficult to determine the appropriate potential at which to take a reading for calibration purposes. A second plateau associated with hypochlorite reduction would be expected outside of this potential window at more cathodic potentials in the oxygen reduction region. Consequently, dissolved oxygen would interfere with the analysis by providing higher currents and thus false positive readings. The equivalent tests were then undertaken in newly prepared standard solutions acidified to $\mathrm{pH} 3$ using $0.1 \mathrm{M} \mathrm{HCl}$. The equivalent LSV scans are presented in figure $6(\mathrm{~B})$. Much higher currents (compared to Figure 6(A)) for similar concentrations were observed. This higher sensitivity allowed clear discrimination between the lower concentration standards. Furthermore, a well-defined plateau region between $02-03 \mathrm{~V}$ was observed enabling facile calibration. Finally, as the Free-chlorine existing entirely as hypochlorous acid, the problems associated with dissolved oxygen as an interferents were removed.
Following the successful demonstration of decreasing $\mathrm{pH}$ increasing chlorine detection sensitivity, the protonator potential established for $\mathrm{pH}$ control in blank water samples was then applied to the initial Free-chlorine standards at $\mathrm{pH} 8.5$. Figure $6(\mathrm{C})$ shows the equivalent scans (to figure $6(\mathrm{~A}))$ performed with the protonator biased at 1.65 V. A significant increase in signal current was observed for each scan performed; similar to Figure 6(B). Figure 6 (D) shows the associated calibration curve fitted using a linear regression technique exhibiting good linearity with a $\mathrm{R}^{2}=$ 0.969 and a measured detection limit of $0.35 \mathrm{ppm}$. Measurements were undertaken in triplicate to determine the error.

\section{5 - Detection of Free-Chlorine in Tap Water Samples:}

To confirm the potential of these sensors for water distribution applications, measurement of residual Free-chlorine were undertaken in tap water samples. For this work, the protonator was again biased at $1.65 \mathrm{~V}$. The sensing electrodes were biased at $1.2 \mathrm{~V}$, to generate the gold oxide, 
and the location of the gold oxide reduction peak observed by scanning cathodically used to confirm $\mathrm{pH} \sim 3.0$ of the solution in the region of the sensing electrode. To account for any matrix effects occurring from the samples, standards were prepared using a stock solution of tap water degassed to remove residual chlorine; confirmed using the commercial DPD method (LOD 0.01 ppm). Hypochlorous acid standards were prepared as before and measurements made in triplicate for each concentration; see figure $7(\mathrm{~A})$. Peak currents, recorded at $0.2 \mathrm{~V}$, were plotted versus each concentration (using the mean of the triplicate measurements) see Figure $7(B)$. Linear regression analysis was again employed to fit a calibration line to the data.
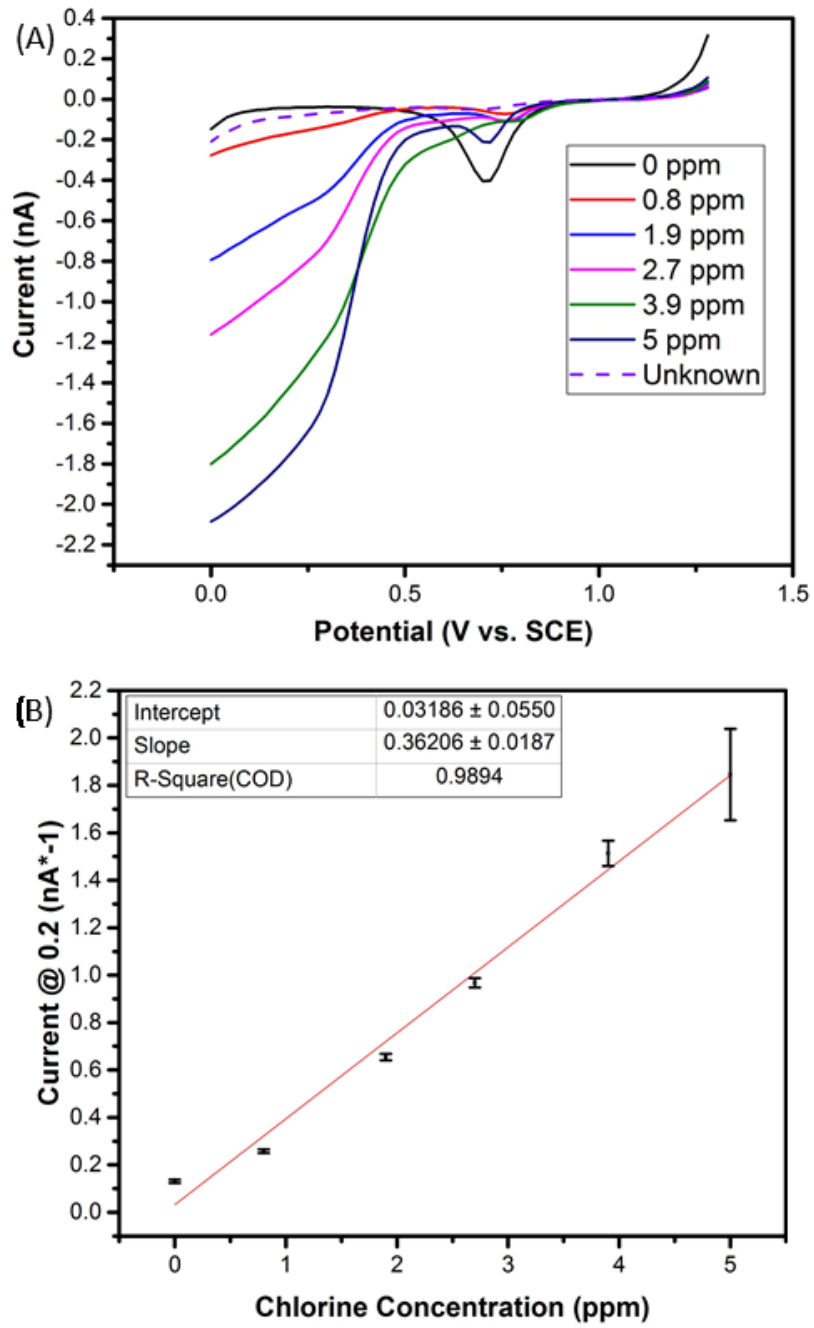

Figure 7: LSV's at gold IDE's from $1.2 \mathrm{~V}$ to $0 \mathrm{~V}$ at a sweep rate of $50 \mathrm{mV} / \mathrm{s}$. (A) Scans of various concentrations of chlorine in tap water. The dashed line represents the unknown sample. (B) Calibration plot for the scans shown in background subtracted (A)

Tap water samples of unknown Free-chlorine concentrations were analysed and the current values, measured at $0.2 \mathrm{~V}$, challenged against the developed calibration curve. A typical LSV recorded for one such sample, dashed line, is presented in Figure $7(\mathrm{~A})$. A Free-chlorine concentration value of $0.33 \mathrm{ppm} \pm 0.02 \mathrm{ppm}$ was determined using the developed electrochemical approach while a commercial
DPD method yielded a concentration of $0.31 \mathrm{ppm}$. In this regard, both methods provided the same result within experimental error thereby confirming the efficacy of the insitu $\mathrm{pH}$ control method.

\section{Conclusions}

We have shown that electrochemical $\mathrm{pH}$ control is an effective approach to detecting residual chlorine concentration in real water samples. Finite element simulations and subsequent electrochemical characterization, using gold interdigitated microelectrode arrays in buffered samples, demonstrated the feasibility of this technique. By designing the sensing electrode to be close to the protonator electrode, the local $\mathrm{pH}$ at the sensing electrode can be tailored to $\mathrm{pH}_{3}$ thereby converting all Free-chlorine into the hypochlorous acid species. Effective chlorine detection was shown in buffered artificial drinking water samples using in-situ $\mathrm{pH}$ control and an enhanced signal response, compared to measurements without $\mathrm{pH}$ control, was demonstrated. Finally, tap water samples were measured using the in-situ $\mathrm{pH}$ control method and the results correlated excellently (within experimental error) with a commercial instrument. This work shows the possibility of an electrochemical approach to reagent-free, in-line sensing of chlorine required for water distribution networks.

\section{AUTHOR INFORMATION}

\section{Corresponding Author \\ *E-mail: alan.oriordan@tyndall.ie}

\section{Author Contributions}

All authors have given approval to the final version of the manuscript.

\section{ACKNOWLEDGMENT}

This publication has emanated in part from research supported by a research grant from Science Foundation Ireland and the Department of Agriculture, Food and Marine on behalf of the Government of Ireland under the Grant 16/RC/3835 (VistaMilk), and supported from research conducted with the financial support of Science Foundation Ireland (SFI) and is cofounded under the European Regional Development Fund under Grant Number 13/RC/2077 (Connect). 


\section{REFERENCES}

M. Szili, I. Kasik, V. Matejec, G. Nagy and B. Kovacs, Sensors and Actuators B: Chemical, 2014, 192, 92-98.

2. Y. Dong, G. Li, N. Zhou, R. Wang, Y. Chi and G. Chen, Anal. Chem., 2012, 84, 8378-8382.

3. T. Lu, L. Zhang, M. Sun, D. Deng, Y. Su and Y. Lv, Anal. Chem., 2016, 88, 3413-3420.

4. A. Staff, Water Chlorination and Chloramination Practices and Principles, 2nd Ed. (M2O), American Water Works Association, 2011.

5. R. D. Morris, A.-M. Audet, I. F. Angelillo, T. C. Chalmers and F. Mosteller, American journal of public health, 1992, 82, 955-963.

6. B. Nemery, P. Hoet and D. Nowak, European Respiratory Journal, 2002.

7. R. L. Jolley, H. Gorchev and D. Hamilton Jr, Water chlorination: environmental impact and health effects. Volume 2, Ann Arbor Science Publishers, Inc., Ann Arbor, MI, 1978.

8. WHO, Monochlorine in Drinking Water, 2004.

9. $\quad$ WHO, $\mathrm{pH}$ in Drinking Water, 2007.

10. F. Arslan, in Nobel Metals, IntechOpen, 2012, DOI: $10.5772 / 32641$.

11. D. L. Harp, Current Technology of Chlorine Analysis for Water and Wastewater, U.S.A., 2002.

12. K. Singh and S. K. Mehta, Analyst, 2016, 141, 2487-2492.

13. V. V. Apyari, M. O. Gorbunova, A. V. Shevchenko, A. A. Furletov, P. A. Volkov, A. V. Garshev, S. G. Dmitrienko and Y. A. Zolotov, Talanta, 2018, 176, 406-411.

14. L. Lu, J. Zhang and X. Yang, Sensors and Actuators B: Chemical, 2013, 184, 189-195.

15. S. Sharma, A. El-Laboudi, M. Reddy, N. Jugnee, S. Sivasubramaniyam, M. El Sharkawy, P. Georgiou, D. Johnston, N. Oliver and A. E. G. Cass, Analytical Methods, 2018, 10, 2088-2095.

16. C. Barrett, K. Dawson, C. O'Mahony and A. O'Riordan, ECS Journal of Solid State Science and Technology, 2015, 4, S3053S3058.

17. I. Seymour, B. O'Sullivan, P. Lovera, J. F. Rohan and A. O'Riordan, presented in part at the 2019 IEEE SENSORS, 2730 Oct. 2019, 2019.

18. D. R. Kumar, S. Kesavan, T. T. Nguyen, J. Hwang, C. Lamiel and J.-J. Shim, Sensors and Actuators B: Chemical, 2017, 240, $818-828$. Fujishima and Y. Einaga, Journal of Electroanalytical Chemistry, 2008, 612, 29-36.

20. F. Kodera, M. Umeda and A. Yamada, Japanese Journal of Applied Physics, 2005, 44, L718-L719. . Kodera, S. Y Kishioka, M. Umeda and A Yamada, Japanese Journal of Applied Physics, 2004, 43, L913-L914.

22. H. H. Girault, Journal of Electroanalytical Chemistry, 2015, 756, 171-178.

23. J. Muñoz, F. Céspedes and M. Baeza, Microchemical Journal, 2015, 122, 189-196.

24. S. Thiagarajan, Z.-Y. Wu and S.-M. Chen, Journal of Electroanalytical Chemistry, 2011, 661, 322-328.

25. O. Ordeig, R. Mas, J. Gonzalo, F. J. Del Campo, F. J. Muñoz and C. de Haro, Electroanalysis, 2005, 17, 1641-1648.

26. T. Soundappan, K. Haddad, S. Kavadiya, R. Raliya and P. Biswas, Applied Nanoscience, 2017, 7, 645-653.

27. R. Olivé-Monllau, J. Orozco, C. Fernández-Sánchez, M. Baeza, J. Bartrolí, C. Jimenez-Jorquera and F. Céspedes, Talanta, 2009, 77, 1739-1744.

28. P. Salazar, M. Martín, J. L. González-Mora and A. R. González-Elipe, Talanta, 2016, 146, 410-416.

29. M. Eryilmaz and I. M. Palabiyik, Tropical Journal of Pharmaceutical Research, 2013, 12, 123-126.

30. G. Lindbergh and D. Simonsson, Journal of The Electrochemical Society, 1990, 137, 3094-3099.

31. T. L. Read, E. Bitziou, M. B. Joseph and J. V. Macpherson, Anal. Chem., 2014, 86, 367-371.

32. J. Rossmeisl, A. Logadottir and J. K. Nørskov, Chemical Physics, 2005, 319, 178-184.

33. K. Dawson, A. Wahl, R. Murphy and A. O'Riordan, The Journal of Physical Chemistry C, 2012, 116, 14665-14673.

34. K. Dawson, A. Wahl, S. Barry, C. Barrett, N. Sassiat, A. J. Quinn and A. O'Riordan, Electrochimica Acta, 2014, 115 , 239-246.

35. S. Barry, K. Dawson, E. Correa, R. Goodacre and A O'Riordan, Faraday discussions, 2013, 164, 283-293.

36. A. J. C. Wahl, I. P. Seymour, M. Moore, P. Lovera, A. O'Riordan and J. F. Rohan, Electrochimica Acta, 2018, 277, 235-243. 